\title{
Squeezing wetting and nonwetting liquids
}

\author{
V. N. Samoilov ${ }^{\text {a) }}$ \\ IFF, FZ-Jülich, 52425 Jülich, Germany and Physics Faculty, Moscow State University, \\ 117234 Moscow, Russia \\ B. N. J. Persson \\ IFF, FZ-Jülich, 52425 Jülich, Germany
}

(Received 22 July 2003; accepted 29 October 2003)

\begin{abstract}
We present molecular-dynamics results for the squeezing of octane $\left(\mathrm{C}_{8} \mathrm{H}_{18}\right)$ between two approaching solid elastic walls with different wetting properties. The interaction energy between the octane bead units and the solid walls is varied from a very small value $(1 \mathrm{meV})$, corresponding to a nonwetting surface with a very large contact angle (nearly 180 degrees), to a high value (18.6 $\mathrm{meV}$ ) corresponding to complete wetting. When at least one of the solid walls is wetted by octane we observe well defined molecular layers develop in the lubricant film when the thickness of the film is of the order of a few atomic diameters. An external squeezing-pressure induces discontinuous, thermally activated changes in the number $n$ of lubricant layers $(n \rightarrow n-1$ layering transitions). With increasing interaction energy between the octane bead units and the solid walls, the transitions from $n$ to $n-1$ layers occur at higher average pressure. This results from the increasing activation barrier to nucleate the squeeze-out with increasing lubricant-wall binding energy (per unit surface area) in the contact zone. Thus, strongly wetting lubricant fluids are better boundary lubricants than the less wetting ones, and this should result in less wear. We analyze in detail the effect of capillary bridge formation (in the wetting case) and droplets formation (in the nonwetting case) on the forces exerted by the lubricant on the walls. For the latter case small liquid droplets may be trapped at the interface, resulting in a repulsive force between the walls during squeezing, until the solid walls come into direct contact, where the wall-wall interaction may be initially attractive. This effect is made use of in some practical applications, and we give one illustration involving conditioners for hair care application. (c) 2004 American Institute of Physics.
\end{abstract} [DOI: $10.1063 / 1.1635813]$

\section{INTRODUCTION}

Tribology, the science of interacting solid surfaces in relative motion, has been studied intensively for many years. It is of great theoretical interest and involves fundamental physics, e.g., questions related to the origin of irreversibility, the role of self-organized criticality, and in the case of boundary lubrication, dynamical phase transitions in molecularly thin lubrication layers. In particular, squeezing of thin lubrication or contamination layers has attracted much attention (see Refs. 1, 2, and 3-5).

In this paper, we consider the lubrication properties of octane $\left(\mathrm{C}_{8} \mathrm{H}_{18}\right)$ confined between two approaching elastic walls with different wetting properties. The interest in squeezing wetting liquids arises from the applications of lubricants to reduce sliding friction and wear. The behavior of nonwetting liquids between rough solid walls is of interest, e.g., in hair care applications. Here conditioners are used to cover the hair fibers with hydrophobic monolayers, which for semi-dry or dry hair reduce the friction and the adhesion between the hair fibers, resulting in easy combing. We have recently presented several computer simulations of boundary lubrication for realistic model systems characterized by different (realistic) parameters (see Refs. 6 and 7-10). For other

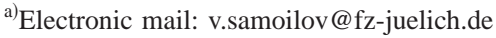

studies involving squeezing of alkanes, see Refs. 11-16.

When two elastic solids with curved, atomically smooth surfaces are squeezed together in a fluid which wets the solid walls, a small contact region is formed, where the surfaces are parallel and separated by an integer number of monolayers of trapped lubricant fluid. For this case it has been shown both experimentally and theoretically that for quasispherical and linear hydrocarbons, the behavior of the lubricant is mainly determined by its interaction with the solid walls that induce layering in the perpendicular direction. ${ }^{3,17-23}$ The thinning of the lubrication film occurs stepwise, by expulsion of individual layers. These layering transitions appear to be thermally activated (see Refs. 24 and 25). Under strong confinement conditions, some lubricant fluids become solidlike. Other fluids, notably water, remain liquidlike up to the last layer that can be removed upon squeezing.

For wetting liquids, the phenomenology of layering transitions in two-dimensional (2D)-solidlike boundary lubrication has been studied in Refs. 6 and 7. We have shown in a series of computer simulations ${ }^{8}$ that the layering transitions are sometimes initiated in solidlike layers by a disordering transition, after which the lubricant behaves in a liquidlike manner for the rest of the squeeze out process.

Simple arguments show that the squeezing of nonwetting liquids occurs according to a different scenario. In this case the fluid molecules interact much stronger with each other 
than with the atoms of the solid walls. Thus, in order to minimize the surface energy, a thin (metastable) liquid film between rough solid walls will break up into small droplets which will be localized in the "large" open spaces between the solid walls, i.e., away from the regions of minimal separation between the walls. Thus in this case no layering transitions will be observed. However, this scenario only holds for small systems with interfacial cavity regions into which the fluid can be transfered. For larger systems where the fluid fills the entire junction between two atomically flat nonwetting surfaces, layering will take place in the confined liquid film. This has in fact been observed in experimental studies by, e.g., Mugele and Salmeron (see Ref. 26).

\section{MODEL}

The model was described in Refs. 7, 8, and 10, but we review it briefly here. We are concerned with the properties of a lubricant film squeezed between the curved surfaces of two elastic solids. In experiments, a system of this type is obtained by gluing two elastic slabs (of thickness $W_{1}$ and $\left.W_{2}\right)$ to "rigid" surface profiles of arbitrary shape. If the radii of curvature of the rigid surfaces are large compared to $W_{1}$ and $W_{2}$, the elastic slabs will deform, reproducing with their free surfaces the (nearly arbitrary) shape of the underlying rigid profiles.

In what follows we denote the lower solid as substrate, which is taken to be fixed in space. The upper solid, denoted as block, will be moving. To account for the elastic response of the slabs, without dealing with the large number of atoms required to simulate a mesoscopic elastic solid we treat explicitly, at the atomistic level, only the last atomic layer of the solids at the interface. These atoms are connected to a rigid curved surface (or profile). The force constants connecting these atoms to the rigid profile, however, are not the bare parameters, determined by the model interatomic potential. Instead, those force constants are treated as effective parameters that implicitly reintroduce the elastic response of the slabs of arbitrary width $W_{1}$ and $W_{2}$.

The model is illustrated in Fig. 1 (see also Refs. 7 and 8). The atoms in the bottom layer of the block (open circles) form a simple square lattice with lattice constant $a$, and lateral dimensions $L_{x}=N_{x} a$ and $L_{y}=N_{y} a$. In the following, periodic boundary conditions are assumed in the $x y$ plane. The atoms interact with each other via "stiff" springs (thick lines) and execute bending and stretching motion characterized by a bending force constant $k_{0 B}$ and a stretching force constant $k_{0}$, respectively. Moreover, each atom is connected to the upper rigid surface profile by "soft" elastic springs (thin lines), of bending force constant $k_{1 B}$ and stretching force constant $k_{1}$. As described in Refs. 7 and 8, the numerical values of all these force constants $k_{0}, k_{0 B}, k_{1}$, and $k_{1 B}$ are determined in such a way as to mimic the elastic response of the entire slab.

The substrate is treated in a similar way as the block, but we use slightly different lattice constant in order to avoid having (low order) commensurate structures formed at the interface. The space between the block and the substrate is occupied by a layer (monolayer or more) of the lubrication fluid (full circles in Fig. 1).

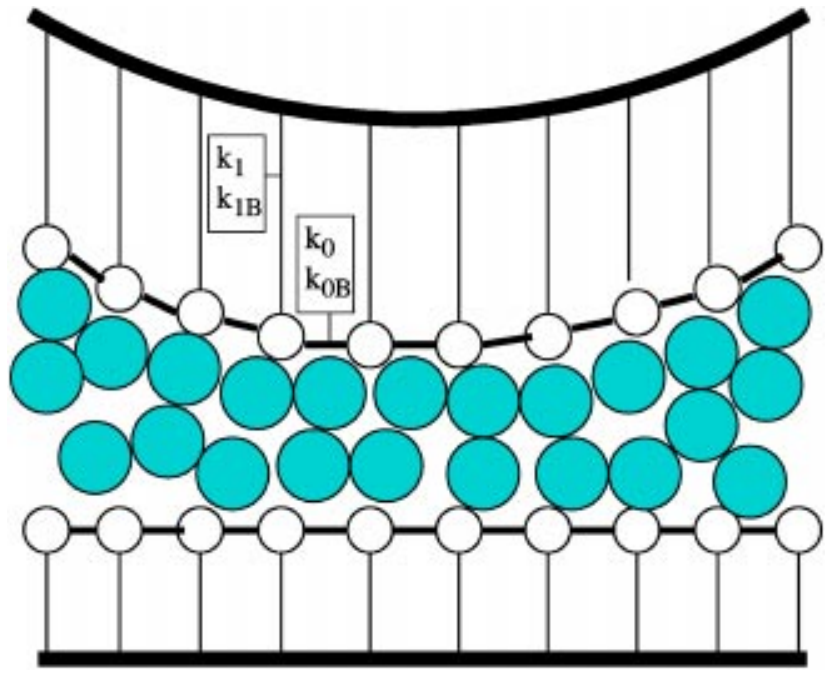

FIG. 1. Schematic picture of the central region of the squeezing model used in the present article.

The MD (molecular dynamics) calculations have been performed by keeping the temperature of the solid walls fixed at their outer boundaries (see Ref. 7). This is a realistic treatment, and it implies that heat flows from the lubricant to the confining walls.

Below we study mainly the average pressure. The pressure acting on a wall atom is defined as the total normal force acting on the wall atom from the lubricant atoms and from the other wall, divided by the area $a^{2}$. The average pressure is the $z$-component of the total force acting on the solid block from the lubricant and the substrate, divided by the total area $L_{x} \times L_{y}$.

Below we provide details of the models used for the block, the substrate and the lubricants in the simulations carried out in the present work, which differ from those described in Refs. 7 and 8.

Both solids the block and the substrate were gold. We used the same elastic modulus and Poisson ratio for the block and substrate, which were $E=7.72 \cdot 10^{10} \mathrm{~Pa}$ and $\nu=0.42$ for gold. We also used the same thickness for the block and substrate $W=50 \AA$. This choice of thicknesses imply that the block and the substrate used in our simulations will deform elastically similar to each other. In the simulations we used a system of lateral dimensions $L_{x}=506 \AA$, $L_{y}=75.9 \AA$. The block rigid profile was taken to be cosine corrugated in the $x$ direction, with corrugation amplitude (difference between maximal and minimal surface heights) $0.1 L_{x}$ and wavelength $L_{x}$. The substrate was atomically flat with $N_{x}=200$ and $N_{y}=30$ atoms in the $x$ and $y$ directions, forming a square lattice with lattice constant $a=2.53 \AA$. The corresponding parameters for the block were $N_{x}=180, N_{y}$ $=27$, and $a=2.81 \AA$.

Octane lubricant $\mathrm{C}_{8} \mathrm{H}_{18}$ was used in the present calculations. It was chosen as having an intermediate chain length (and properties) among the linear alkanes of different chain lengths $\mathrm{C}_{3} \mathrm{H}_{8}, \mathrm{C}_{4} \mathrm{H}_{10}, \mathrm{C}_{8} \mathrm{H}_{18}, \mathrm{C}_{9} \mathrm{H}_{20}, \mathrm{C}_{10} \mathrm{H}_{22}, \mathrm{C}_{12} \mathrm{H}_{26}$ and $\mathrm{C}_{14} \mathrm{H}_{30}$, lubricating properties of which have been considered recently in Ref. 10 . We considered $\mathrm{C}_{8} \mathrm{H}_{18}$ chain molecules consisting of eight beads in the united atom represen- 
tation. The Lennard-Jones potential was used to model the interaction between beads of different chains

$$
v(r)=4 \epsilon_{0}\left[\left(\frac{r_{0}}{r}\right)^{12}-\left(\frac{r_{0}}{r}\right)^{6}\right],
$$

and the same potential with modified parameters $\left(\epsilon_{1}, r_{1}\right)$ and $\left(\epsilon_{2}, r_{2}\right)$ was used for the interaction of each bead with the substrate and block atoms, respectively. For the interactions within the $\mathrm{C}_{8} \mathrm{H}_{18}$ we used the OPLS model (Refs. 27 and 28 , including flexible bonds, bond bending and torsion interaction, which results in bulk properties in good agreement with experimental data. The parameters were $\epsilon_{0}=5.12 \mathrm{meV}$ for the both interior and end beads and $r_{0}=3.905 \AA$ in all cases. Atomic mass 14 (for interior $\mathrm{CH}_{2}$ beads) and 15 (for the $\mathrm{CH}_{3}$ end groups) were used. For the interaction of each bead with the substrate and block atoms we took $r_{1}=r_{2}$ $=3.28 \AA .{ }^{29}$ To study the lubricating properties of octane confined between two approaching solid walls with different wetting properties we changed the interaction energy between the octane bead units and the solid walls ( $\epsilon_{1}$ and $\epsilon_{2}$ ), from a very small value $(1 \mathrm{meV})$ corresponding to a nonwetting surface with a very large contact angle (nearly 180 degrees) to a high value $(18.6 \mathrm{meV})^{29}$ corresponding to complete wetting. The latter choice reflects the stronger interaction between the beads and metal surfaces than between the bead units of different lubricant molecules.

Within a $\mathrm{C}_{8} \mathrm{H}_{18}$ chain we assume nearest neighbor $\mathrm{C}$ atoms are connected via springs with the spring constant $k$, which was chosen equal to $10 \mathrm{~N} / \mathrm{m}$. Time step was equal to 1 fs. We used an angle bending interaction of the form $E(\cos \theta) / k_{B}=\frac{1}{2} k_{\text {bend }}\left(\cos \theta-\cos \theta_{0}\right)^{2}$ with $k_{\text {bend }}=62543 \mathrm{~K}$ and $\theta_{0}=2.0001 \mathrm{rad}^{28}$ For the dihedral interaction we used the functional form in term of a cosine Fourier series $E(\phi) / k_{B}$ $=\sum_{i=0}^{3} c_{i} \cos ^{i}(\phi) \quad$ with parameters $c_{0}=1009.99 \mathrm{~K}, \quad c_{1}$ $=2018.95 \mathrm{~K}, c_{2}=136.37 \mathrm{~K}, c_{3}=-3165.30 \mathrm{~K} .{ }^{28}$ Internal beads of separation greater than three units were treated similarly as beads from different chains.

For interaction between atoms of the block and the substrate we used the Lennard-Jones potential with the parameters $\epsilon_{12}=18.6 \mathrm{meV}$ and $r_{12}=3.28 \AA$, the same as used in Ref. 10 .

By variation of the interaction energy between the octane bead units and the solid walls $\left(\epsilon_{1}\right.$ and $\left.\epsilon_{2}\right)$ it was possible to examine similarities and differences between squeezing of lubricants for different wetting of the walls. We considered both symmetric pair of parameters $\epsilon_{1}=\epsilon_{2}$ and asymmetric pair of parameters $\left(\epsilon_{1}\right.$ varied and $\epsilon_{2}$ $=18.6 \mathrm{meV}$ ).

\section{SIMULATION RESULTS AND DISCUSSION}

We now describe the results obtained from our simulations for octane $\mathrm{C}_{8} \mathrm{H}_{18}$ squeezed between two solid elastic walls with different wetting properties. Thus, we have changed the interaction energy between the octane bead units and the solid walls, from a very small value $(1 \mathrm{meV})$ corresponding to a nonwetting surface with a very large contact angle [nearly 180 degrees, see Fig. 2(a)], to a high value (18.6 meV) corresponding to complete wetting, see Fig. 2(d). (a)

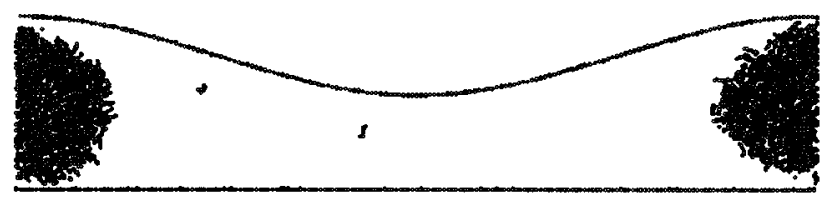

(b)

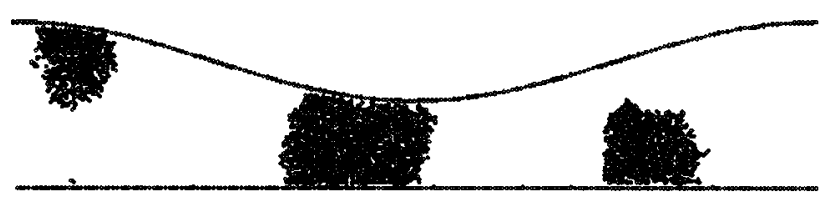

(c)

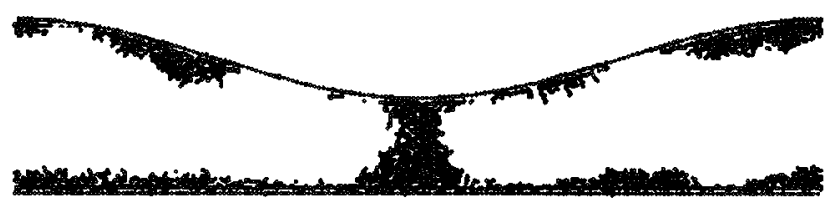

(d)

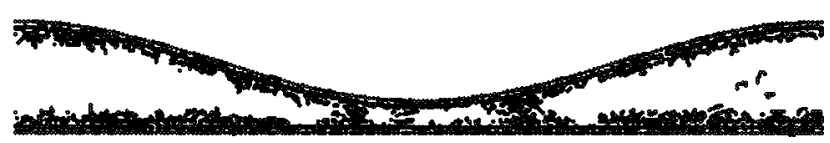

FIG. 2. The nature of the octane $\mathrm{C}_{8} \mathrm{H}_{18}$ film between two solid elastic walls, for four different cases corresponding to nonwetting [cases (a) and (b)] and wetting [cases (c) and (d)] octane liquid. Symmetric pair of the bead-wall atom interaction energy parameters $\epsilon_{1}=\epsilon_{2}$ was used here, equal to (a) 1 $\mathrm{meV}$, (b) $3 \mathrm{meV}$, (c) $8 \mathrm{meV}$, and (d) $18.6 \mathrm{meV}$.

The octane films for the intermediate bead-wall interaction energy parameters $\epsilon_{1}=\epsilon_{2}$, equal to 3 and $8 \mathrm{meV}$, are presented in Figs. 2(b) and 2(c), respectively.

Figure 3 shows the variation of the average pressure during squeezing, as the block moves a distance of $24 \AA$ toward the substrate. The squeeze velocity was $v_{z}=2 \mathrm{~m} / \mathrm{s}$. A few test calculations at lower squeeze velocity gave very similar results as for $v_{\mathrm{z}}=2 \mathrm{~m} / \mathrm{s}$ indicating that no new physics may be expected with lower squeeze velocities, but the layering transitions occur at slightly lower squeezing pressures due to the fact that the nucleation of squeeze-out is a stochastic thermally activated process. We used the same bead-wall interaction energy parameters for both solid walls, $\epsilon_{1}=\epsilon_{2}$, equal to (a) $1 \mathrm{meV}$, (b) $3 \mathrm{meV}$, (c) $8 \mathrm{meV}$, (d) $12 \mathrm{meV}$, and (e) 18.6 $\mathrm{meV}$. For the systems exhibiting complete wetting (zero contact angle), namely cases (c)-(e), $n \rightarrow n-1$ ( $n=3,2,1)$ layering transitions are observed and indicated in the figure. For the nonwetting systems [cases (a) and (b)] no layering transitions can be observed, and, in fact, no fluid occurs in the region between the solid walls where the spacing is the smallest. (This scenario only holds for small systems with interfacial cavity regions into which the fluid can be transfered. For larger systems where the fluid fills the entire junction between two atomically flat nonwetting surfaces, layering may take place in the confined liquid film.) This is illustrated by the snapshot pictures shown in Fig. 4 for case (a), taken at the separations corresponding to the vertical 


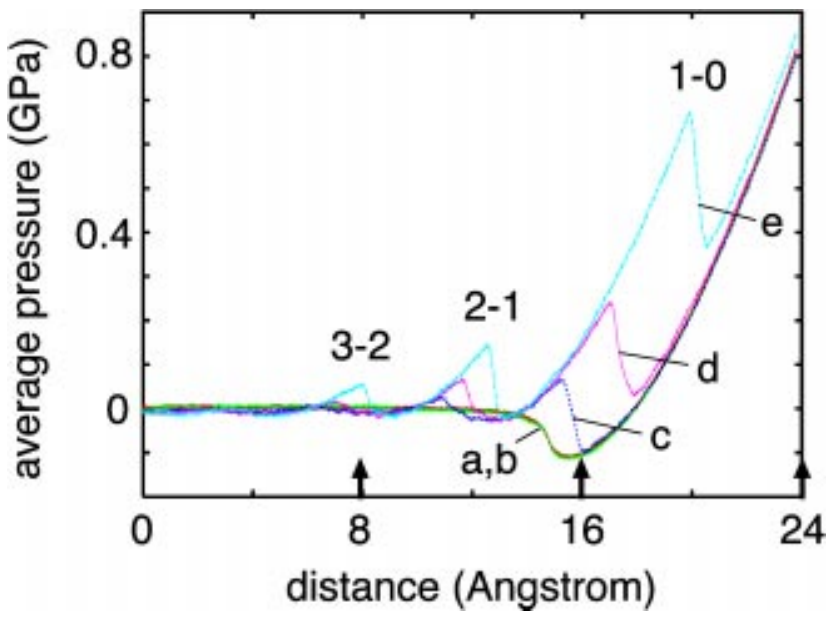

FIG. 3. The variation of the average pressure during squeezing developed as the block moves a distance of $24 \AA$ toward the substrate. Octane $\mathrm{C}_{8} \mathrm{H}_{18}$ was used as lubricant. Squeeze velocity was $v_{\mathrm{z}}=2 \mathrm{~m} / \mathrm{s}$. Symmetric pair of parameters $\epsilon_{1}=\epsilon_{2}$ was used here, equal to (a) $1 \mathrm{meV}$, (b) $3 \mathrm{meV}$, (c) $8 \mathrm{meV}$, (d) $12 \mathrm{meV}$, and (e) $18.6 \mathrm{meV}$. The $n \rightarrow n-1$ layering transitions are observed for three latter systems. The arrows indicate the block displacements at the snapshot pictures presented in Figs. 4 and 5 for cases (a) and (e), respectively.

arrows in Fig. 3. In contrast, for fluids which wet the solid walls, the fluid tends to accumulate in the region where the wall-wall separation is the smallest, forming a capillary bridge. This is illustrated in Figs. 2(d) and 5 for case (e) in Fig. 3. The snapshot pictures correspond again to the block displacements $d=8,16$, and $24 \AA$, as indicated by the vertical arrows in Fig. 3.

Figure 2(a) shows that an octane liquid, when the spacing between the solid walls is large enough, forms a nearly spherical droplet in case (a). When the liquid is confined between closely spaced solid walls, there is not enough space for the droplet to take this ideal form. In order to minimize the surface energy the octane liquid is localized to the large open space between the solid walls (see Fig. 4). Since the surface energy of the liquid droplet decreases as the separa-

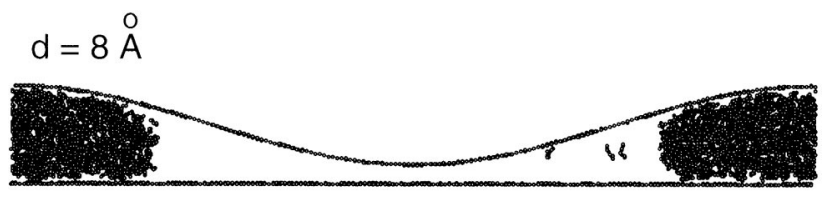

$$
d=16 \stackrel{\circ}{\AA}
$$

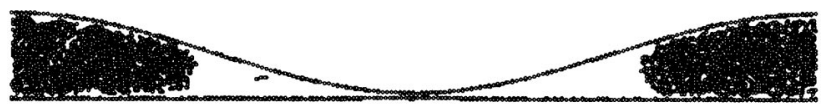

$$
d=24 \stackrel{\circ}{\AA}
$$

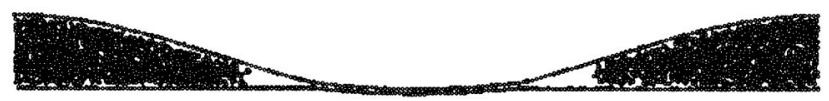

FIG. 4. Snapshot pictures for the approach of a cosine corrugated block toward a flat substrate with octane $\mathrm{C}_{8} \mathrm{H}_{18}$ as lubricant. Squeeze velocity was $v_{\mathrm{z}}=2 \mathrm{~m} / \mathrm{s}$. Parameters $\epsilon_{1}=1 \mathrm{meV}$ and $\epsilon_{2}=1 \mathrm{meV}$ were used here. The snapshot pictures correspond to the block displacements $d=8,16$, and $24 \AA$. Nonwetting behavior is clearly seen.

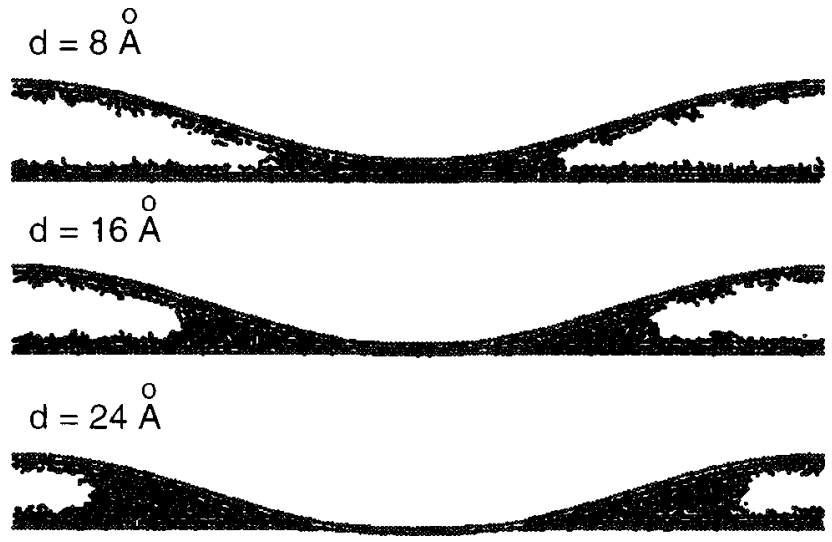

FIG. 5. Snapshot pictures for the approach of a cosine corrugated block toward a flat substrate with octane $\mathrm{C}_{8} \mathrm{H}_{18}$ as lubricant. Squeeze velocity was $v_{\mathrm{z}}=2 \mathrm{~m} / \mathrm{s}$. Parameters $\epsilon_{1}=18.6 \mathrm{meV}$ and $\epsilon_{2}=18.6 \mathrm{meV}$ (Ref. 29) were used here. The same parameters were also used in our recent papers (Refs. 8 and 10). The snapshot pictures correspond to the block displacements $d$ $=8,16$, and $24 \AA$. Wetting behavior is clearly seen and we observe $n \rightarrow n$ -1 layering transitions.

tion between the solid walls increases (since the droplet can now take a more spherical shape), the droplet will exert a repulsive force on the solid walls. Thus, as long as the direct (attractive) wall-wall interaction can be neglected, the average force exerted on the walls is repulsive. This is illustrated in Fig. 6 [curve (a)] (a magnified section from Fig. 3), which shows the average pressure acting on the block (or the substrate) as a function of the displacement of the block toward the substrate. Curve (b) in the same figure shows the (average) pressure acting on the block for the case of complete wetting, corresponding to case (e) in Fig. 3. In this case a capillary bridge is formed between the solid walls [see Fig. $2(d)]$ resulting in a negative pressure acting on the block. Note that the magnitude of the negative pressure is about five

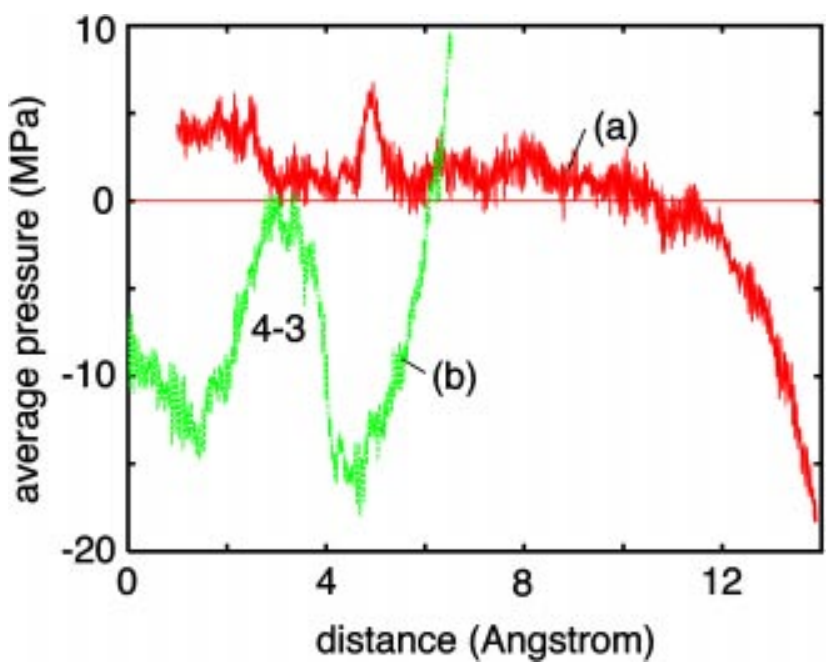

FIG. 6. The average pressure acting on the walls for (a) a nonwetting liquid, and (b) a wetting liquid. For octane with $\epsilon_{1}=\epsilon_{2}$, equal to $1 \mathrm{meV}$ and 18.6 $\mathrm{meV}$, respectively. 
times higher than the positive pressure for the nonwetting case ( $-10 \mathrm{MPa}$ as compared to $\sim 2 \mathrm{MPa}$ ). The "pressure peak" observed for the wetting liquid at the block displacement $d \approx 3 \AA$ in Fig. 6 [curve (b)], corresponds to the $n=4$ $\rightarrow 3$ layering transition, and the strong increase in average pressure around a distance $d \sim 6 \AA$ is due to the pressure buildup before the onset of the $n=3 \rightarrow 2$ layering transition, as is clearly seen in Fig. 3 [curve (e)].

From the discussion above it is clear that if a nonwetting liquid is squeezed between solid walls with roughness (either geometrical or chemical), small liquid droplets may be trapped at the interface [see Fig. 7(a)], resulting in a repulsive force exerted on the walls during squeezing, until the solid walls come into direct contact, where the wall-wall interaction may be initially attractive. This effect is made use of in some practical applications, and we will give one illustration below involving conditioners for hair care application.

For a nonwetting liquid, when the solid walls come into direct contact the wall-wall interaction is initially attractive, which results in a negative pressure acting on the block. This is clearly seen in Fig. 6 [curve (a)] at distances $d>12 \AA$ and in Fig. 3 [curve (a)] around a distance $d \sim 16 \AA$. This corresponds to the system configuration shown in Fig. 4 (see snapshot picture taken at the block displacement $d=16 \AA$ ).

Figure 5 shows three snapshot pictures under the same

(a)

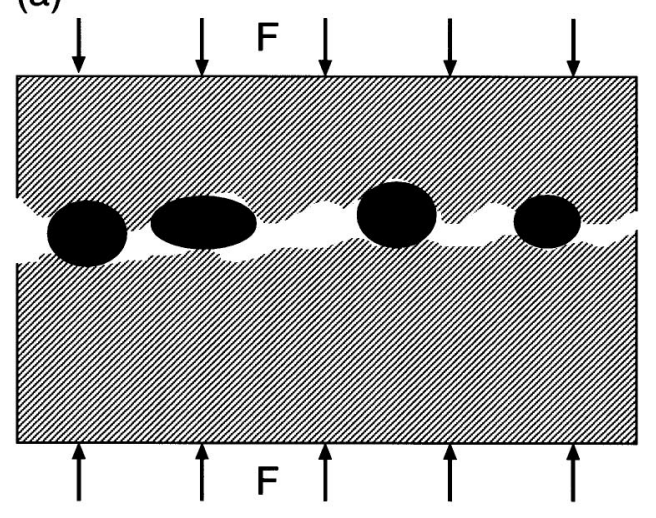

(b)

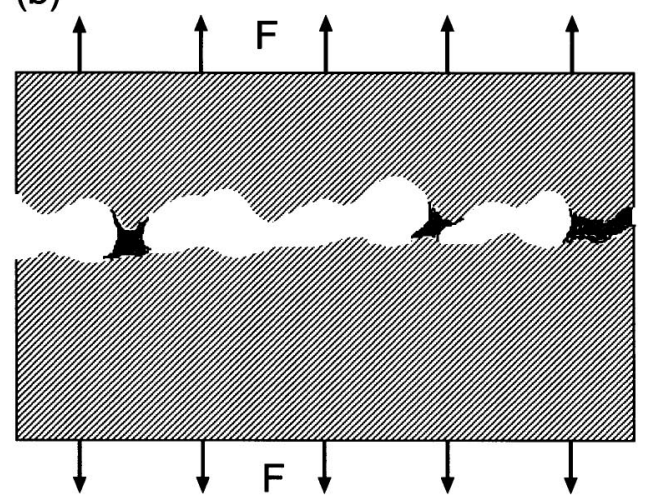

FIG. 7. (a) A nonwetting liquid trapped between two solid walls gives rise to an effective repulsion between the walls. (b) Capillary bridges formed by a wetting liquid. In this case the liquid gives rise to attraction between the walls.

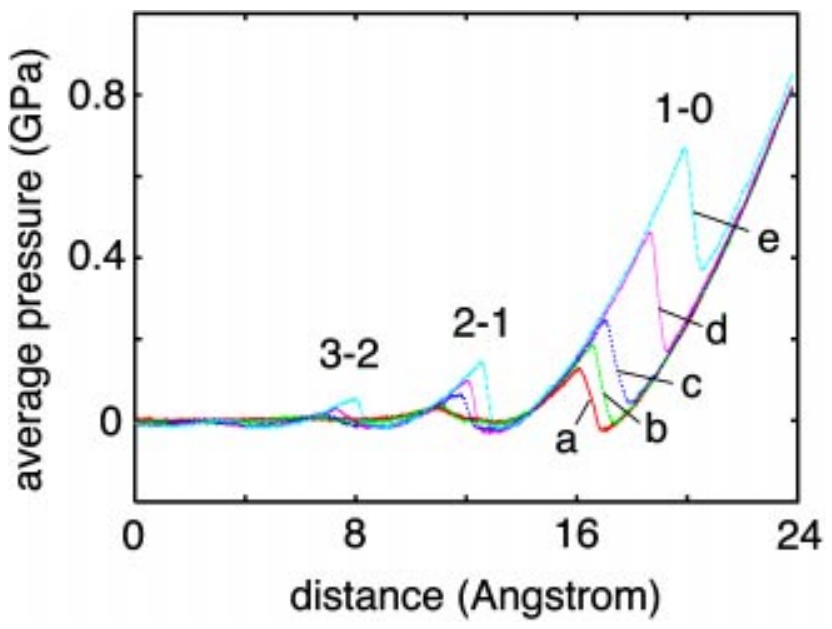

FIG. 8. The variation of the average pressure during squeezing as the block moves a distance of $24 \AA$ toward the substrate. Octane $\mathrm{C}_{8} \mathrm{H}_{18}$ was used as lubricant. Squeeze velocity was $v_{\mathrm{z}}=2 \mathrm{~m} / \mathrm{s}$. Asymmetric pair of parameters $\epsilon_{1}$ and $\epsilon_{2}$ was used here with $\epsilon_{1}$ equal to (a) $1 \mathrm{meV}$, (b) $3 \mathrm{meV}$, (c) $8 \mathrm{meV}$, (d) $12 \mathrm{meV}$, and (e) $18.6 \mathrm{meV}$ and $\epsilon_{2}=18.6 \mathrm{meV}$ for all cases. The $n \rightarrow n$ -1 layering transitions are observed for all the systems.

conditions as in Fig. 4, but now for a stronger interaction between the octane bead units and the solid walls. Thus, we have used $\epsilon_{1}=\epsilon_{2}=18.6 \mathrm{meV}$, which results in octane wetting the solid walls [see Fig. 2(d)]. The same parameters were also used in our recent papers. ${ }^{8,10}$ In this case, as the solid walls (covered with the wetting octane liquid) first come into contact, a strong attraction occurs from the formation of capillary bridges between the walls. This is illustrated in Fig. 7(b) for the case of a randomly rough surface. In this case the lubricant molecules form layers parallel to the solid surfaces which are squeezed out in a quantized manner as discussed in detail elsewhere (see, for instance, Refs. 7, 8, and 10). Immediately before the onset of squeeze out, the average pressure is (almost) always positive (repulsion) and takes higher and higher values as the film thickness decreases, see Fig. 3 [curve (e)].

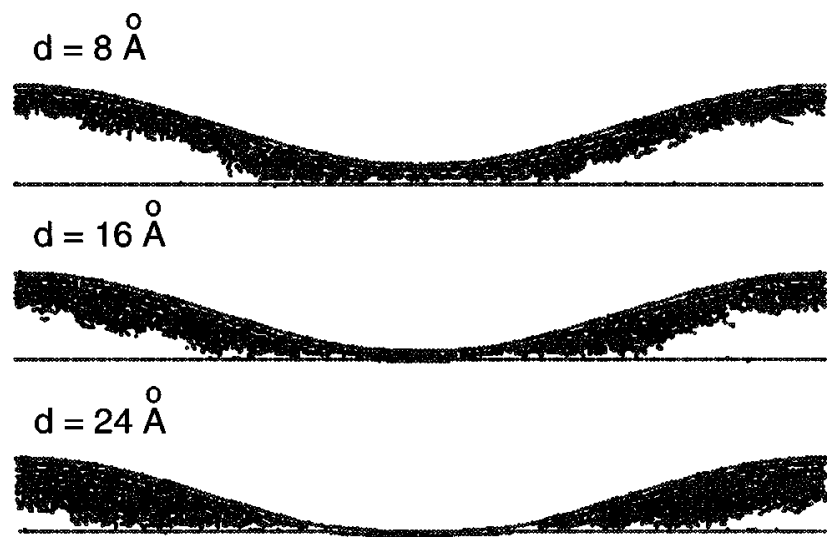

FIG. 9. Snapshot pictures for the approach of a cosine corrugated block toward a flat substrate with octane $\mathrm{C}_{8} \mathrm{H}_{18}$ as lubricant. Squeeze velocity was $v_{\mathrm{z}}=2 \mathrm{~m} / \mathrm{s}$. Parameters $\epsilon_{1}=1 \mathrm{meV}$ and $\epsilon_{2}=18.6 \mathrm{meV}$ were used here. The snapshot pictures correspond to the block displacements $d=8,16$, and $24 \AA$. For this asymmetric pair of parameters one deserves nonwetting behavior of lubricant at the surface of the substrate and wetting behavior at the surface of the block. 


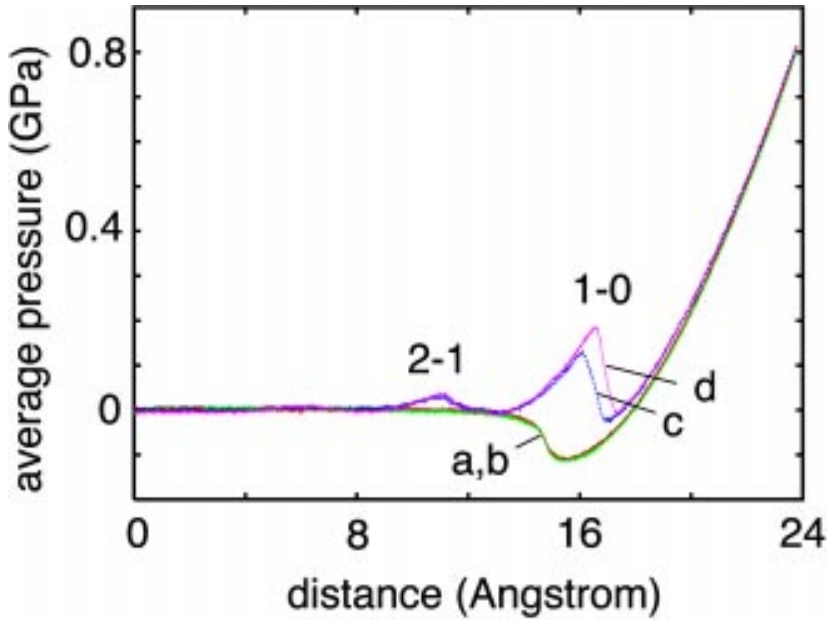

FIG. 10. Direct comparison of the variation of the average pressure during squeezing as the block moves a distance of $24 \AA$ toward the substrate for symmetric $\left(\epsilon_{1}=\epsilon_{2}\right)$ and asymmetric pairs of parameters. Octane $\mathrm{C}_{8} \mathrm{H}_{18}$ was used as lubricant. Squeeze velocity was $v_{\mathrm{z}}=2 \mathrm{~m} / \mathrm{s}$. Symmetric pair of parameters $\epsilon_{1}=\epsilon_{2}$ was used equal to (a) $1 \mathrm{meV}$ and (b) $3 \mathrm{meV}$, and asymmetric pair of parameters with $\epsilon_{1}$ equal to (c) $1 \mathrm{meV}$ and (d) $3 \mathrm{meV}$, and $\epsilon_{2}$ $=18.6 \mathrm{meV}$ for both cases (c) and (d). The $n \rightarrow n-1$ layering transitions are observed for two latter systems.

Figure 8 shows the variation of the average pressure during squeezing under the same conditions as in Fig. 3, except that the bead-wall interaction now is asymmetric with $\epsilon_{1}$ equal to (a) $1 \mathrm{meV}$, (b) $3 \mathrm{meV}$, (c) $8 \mathrm{meV}$, (d) $12 \mathrm{meV}$, and (e) $18.6 \mathrm{meV}$, and with $\epsilon_{2}=18.6 \mathrm{meV}$ for all cases. In these cases, $n \rightarrow n-1(n=3,2,1)$ layering transitions are observed for all the systems. In Fig. 9 we show snapshot pictures for case (a) when the block has been displaced toward the substrate by 8,16 , and $24 \AA$. For this asymmetric pair of parameters, nonwetting of the substrate surface and wetting of the block are clearly seen.

In Fig. 10 we compare the variation of the average pressure during squeezing for symmetric $\left(\epsilon_{1}=\epsilon_{2}\right)$ and asymmetric pairs of parameters. In the symmetric case we used $\epsilon_{1}$ $=\epsilon_{2}$ equal to (a) $1 \mathrm{meV}$ and (b) $3 \mathrm{meV}$, and for the asymmetric pair of parameters we used $\epsilon_{1}$ equal to (c) $1 \mathrm{meV}$ and (d) $3 \mathrm{meV}$, and $\epsilon_{2}=18.6 \mathrm{meV}$ for both cases (c) and (d). The $n \rightarrow n-1$ layering transitions are observed for two latter systems.

Finally, we note that the influence of wetting and nonwetting liquids on adhesion is paradoxical. Thus, when contacting solids are completely surrounded by a wetting liquid, the bond between the surfaces may be broken, while a nonwetting liquid may strengthen the bond. However, when a small amount (e.g., a molecular thick film) of liquid occurs at the interface between the solids, it may have the opposite effect (see above). This fact is often made use of in nature, and in engineering applications. Thus, for example, some insects inject a very thin film of a wetting liquid at the interface between the attachment organs and the (usually rough) substrate in order to increase adhesion. On the other hand, in hair care applications the hair fibers are covered by thin (monolayer) hydrophobic coating, which results in effective repulsion between semi-wet (or moist) hair fibers, see below. Another well-known example is the influence of water on the adhesion between sand particles: Because of surface roughness, the adhesion between dry sand particles is usually very small. However, moist sand particles can adhere with measurable strength, as utilized by children when building sandcastles (note: Sand particles usually have polar surfaces which are wetted by water). Finally, when sand is completely wet (i.e., immersed in water) the adhesion is again very low.

\section{AN APPLICATION: CONDITIONER AND THE COMBING OF WET HAIR}

The surfaces of clean hair fibers in water at a $\mathrm{pH}$ larger than $\sim 4$ have negatively charged groups. ${ }^{30}$ Thus when the fibers are fully immersed in water one would expect repulsive Coulomb forces to occur between the fibers, which tend to keep them separated from each other, similar to the case of a negatively charged rubber surface in contact with a (negatively charged) glass surface, where a thin liquid layer can be trapped between the surfaces even at squeezing pressures as high as $\sim 0.1 \mathrm{MPa}$. Thus, completely wet hair may be relatively easy to comb. However, for semi-dry hair, as may result from drying the hair with a towel, the situation is different: Since the contact angle for water on the clean, negatively charged, hair fibers is zero (complete wetting), capillary bridges will form in the contact areas between the hair fibers, see Fig. 11(b). The pressure in the capillary bridges is much lower than the surrounding atmospheric pressure, leading to strong effective adhesion between the fibers. During combing of semi-dry hair, the liquid bridges will be elongated before they break, leading to an effective long-range and strong attraction between the fibers, resulting in very bad combing properties. Conditioners for hair care applications are used to reduce the combing force. These fluids contain molecules with a long hydrocarbon chain, and a positively charged head group, which bind strongly to the negatively charged groups on the hair fibers forming a grafted monolayer film, with the inert hydrocarbon chains pointing into the water, see Fig. 12. The coated hair fibers are hydrophobic with large contact angle for water. ${ }^{30}$ Thus, the thin water film, which may be left on the hair fibers after drying the hair with a towel, will immediately break up into small water droplets (dewetting transition). If no pinning by defects would occur the small droplets would tend to join together into larger droplets, but due to surface defects on the hair fibers (e.g., small areas not covered by the hydrophobic monolayer film), one may expect that most of the droplets would remain pinned at different locations along the hair fibers, see Fig. 11(c). When two hair fibers come into contact without an external squeezing force, they will be separated by a small but finite distance determined by the (average) radius of the water droplets and by the contact angle, see Fig. 11(d). If the fibers are squeezed toward each other, the droplets will deform as indicated in Fig. 11(e). (We assume here that the droplet positions are pinned so that they do not rotate away from the region between the hair fibers.) Since the pressure inside the droplets is much higher than the atmospheric pressure, and since the contact area between the droplet and the hair fibers increases during squeezing, the droplets will exert a strong repulsive force on the hair fibers. Thus, in this case the hair fibers tend to be separated from 


\section{Wetting}

(a)

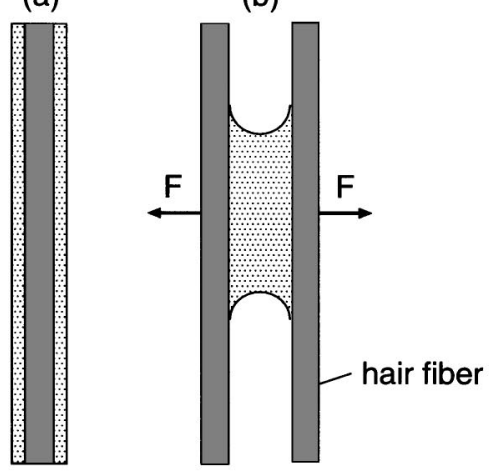

\section{Nonwetting}

(c)
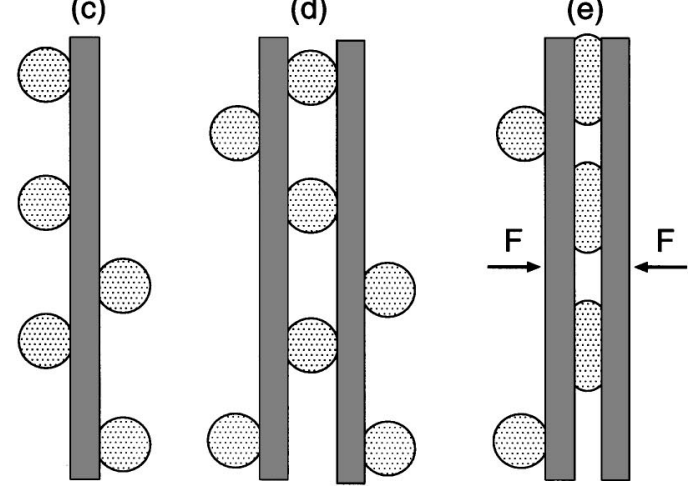

FIG. 11. The interaction between wet hair fibers. (a) Natural (uncoated) hair fibers are hydrophilic, and coated by thin water films. (b) When two fibers come into contact, a liquid bridge is formed leading to an effective attractive interaction between the fibers. In the absence of external forces direct fiberfiber contact will occur (not shown). (c) If the hair fibers are coated by hydrophobic monolayer films (see Fig. 12), the water film will break up into small droplets (dewetting transition) with nonzero contact angles determined by the interface energies (Young's law). In this case, in the absence of external forces, there is a finite natural separation between two hair fibers [see (d)]. (e) At separation shorter than the natural separation, the fibers will repel each other.

each other during combing, leading to very small fiber-fiber adhesion and friction, and good combing properties. In addition, owing to the inert nature of the monolayer film shown in Fig. 12, even in the absence of water the adhesion and

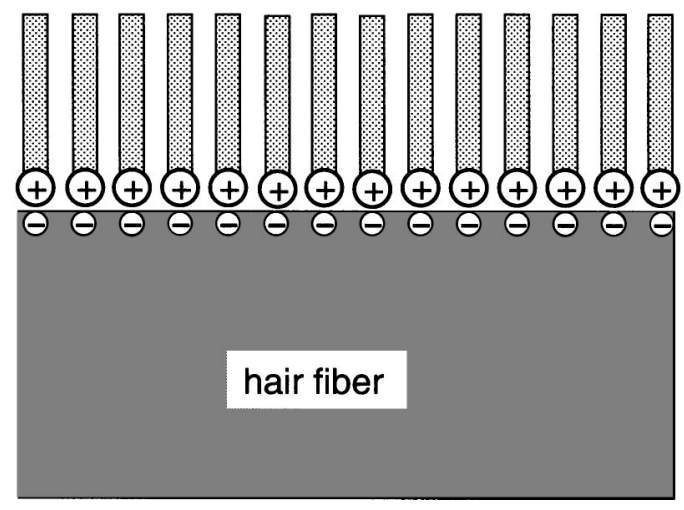

FIG. 12. Coulomb attraction between the negatively charged hair surface and the positive charged head groups of the conditioner molecules causes head-down/tail-up adsorption at the interface. friction between the hair fibers is reduced for hair treated with a conditioner, as compared to untreated hair.

\section{SUMMARY AND CONCLUSION}

We have studied the lubricating properties of octane $\left(\mathrm{C}_{8} \mathrm{H}_{18}\right)$ confined between two approaching solid elastic walls with different wetting properties. We have changed the interaction energy between the octane bead units and the solid walls, from a very small value $(1 \mathrm{meV})$, corresponding to a nonwetting surface with a very large contact angle (nearly 180 degrees), to a high value $(18.6 \mathrm{meV})$ corresponding to complete wetting. The results indicate that when at least one of the solid walls is wetted by octane, well defined molecular layers develop in the lubricant film when the width of the film is of the order of a few atomic diameters. An external squeezing-pressure induces discontinuous, thermally activated changes in the number $n$ of lubricant layers ( $n \rightarrow n-1$ layering transitions). We find that with increasing lubricant molecule-wall binding energy, the transitions from $n$ to $n-1$ layers occur at higher average pressure; this results from the higher activation barrier to nucleate the squeeze-out with increasing lubricant-wall binding energy per unit area. Thus, the more wetting lubricant fluids are better boundary lubricants than the less wetting ones, and this should result in less wear.

We analyzed the effect of capillary bridge formation (in the wetting case) and droplets formation (in the nonwetting case) on the forces exerted by the lubricant on the walls. For the latter case small liquid droplets may be trapped at the interface, resulting in a repulsive force exerted on the walls during squeezing, until the solid walls come into direct contact, where the wall-wall interaction may be initially attractive. This effect is made use of in some practical applications, and we have presented one illustration involving conditioners for hair care application.

\section{ACKNOWLEDGMENTS}

One of the authors (V.N.S.) acknowledges support from IFF, FZ-Jülich, hospitality and help of the staff during his research visits. B.N.J.P. thanks the EC for a "Smart Quasicrystals" grant under the EC Program "Promoting Competitive and Sustainable Growth." B.N.J.P. thanks Dr. K. Mathauer (BASF Aktiengesellschaft) for useful discussions.

${ }^{1}$ B. N. J. Persson, Sliding Friction: Physical Principles and Applications (Springer, Heidelberg, 2000).

${ }^{2}$ B. N. J. Persson, Surf. Sci. Rep. 33, 83 (1999).

${ }^{3}$ J. N. Israelachvili, Intermolecular and Surface Forces (Academic, London, 1995).

${ }^{4}$ J. Krim, Sci. Am. 275, 74 (1996).

${ }^{5}$ J. Krim, Surf. Sci. 500, 741 (2002).

${ }^{6}$ B. N. J. Persson and P. Ballone, Solid State Commun. 115, 599 (2000).

${ }^{7}$ B. N. J. Persson and P. Ballone, J. Chem. Phys. 112, 9524 (2000).

${ }^{8}$ B. N. J. Persson, V. N. Samoilov, S. Zilberman, and A. Nitzan, J. Chem. Phys. 117, 3897 (2002).

${ }^{9}$ B. N. J. Persson, O. Albohr, F. Mancosu, V. Peveri, V. N. Samoilov, and I. M. Sivebaek, Wear 254, 835 (2003).

${ }^{10}$ I. M. Sivebaek, V. N. Samoilov, and B. N. J. Persson, J. Chem. Phys. 119, 2314 (2003)

${ }^{11}$ U. Landman, W. D. Luedtke, and E. M. Ringer, Wear 153, 3 (1992).

${ }^{12}$ U. Landman, W. D. Luedtke, J. Ouyang, and T. K. Xia, J. Appl. Phys. 32, 1444 (1993). 
${ }^{13}$ U. Landman and W. D. Luedtke, Appl. Surf. Sci. 92, 237 (1996).

${ }^{14}$ J. Gao, W. D. Luedtke, and U. Landman, J. Chem. Phys. 106, 4309 (1997).

${ }^{15}$ J. Gao, W. D. Luedtke, and U. Landman, J. Phys. Chem. B 101, 4013 (1997).

${ }^{16}$ S. T. Cui, P. T. Cummings, and H. D. Cochran, J. Chem. Phys. 114, 7189 (2001).

${ }^{17}$ M. L. Gee, P. M. McGuiggan, J. N. Israelachvili, and A. M. Homola, J. Chem. Phys. 93, 1895 (1990).

${ }^{18}$ J. P. Gao, W. D. Luedtke, and U. Landman, Phys. Rev. Lett. 79, 705 (1997).

${ }^{19}$ H. Tamura, M. Yoshida, K. Kusakabe, C. Young-Mo, R. Miura, M. Kubo, K. Teraishi, A. Chatterjee, and A. Miyamoto, Langmuir 15, 7816 (1999).

${ }^{20}$ A. L. Demirel and S. Granick, Phys. Rev. Lett. 77, 2261 (1996).
${ }^{21}$ A. L. Demirel and S. Granick, J. Chem. Phys. 109, 6889 (1998).

${ }^{22}$ J. Klein and E. Kumacheva, Science 269, 816 (1995).

${ }^{23}$ E. Kumacheva and J. Klein, J. Chem. Phys. 108, 7010 (1998).

${ }^{24}$ B. N. J. Persson and E. Tosatti, Phys. Rev. B 50, 5590 (1994).

${ }^{25}$ B. N. J. Persson, Chem. Phys. Lett. 324, 231 (2000).

${ }^{26}$ F. Mugele and M. Salmeron, Phys. Rev. Lett. 84, 5796 (2000).

${ }^{27}$ W. L. Jorgensen, J. D. Madura, and C. J. Swenson, J. Am. Chem. Soc. 106, 6638 (1984).

${ }^{28}$ D. K. Dysthe, A. H. Fuchs, and B. Rousseau, J. Chem. Phys. 112, 7581 (2000).

${ }^{29}$ T. K. Xia, J. Ouyang, M. W. Ribarsky, and U. Landman, Phys. Rev. Lett. 69, 1967 (1992).

${ }^{30}$ R. Y. Lochhead, Cosmet. Toiletries 103, 23 (1988). 